Annuaire suisse de politique de développement

27-2 | 2008

Migration et développement: un mariage arrangé

\title{
Eclairage - Des cafés littéraires de Bagdad aux cafés virtuels
}

Diane Duclos

\section{(2) OpenEdition}

1 Journals

Édition électronique

URL : http://journals.openedition.org/aspd/171

DOI : 10.4000/aspd. 171

ISSN : 1663-9669

Éditeur

Institut de hautes études internationales et du développement

Édition imprimée

Date de publication : 1 décembre 2008

Pagination : 69-72

ISBN : 978-2-940415-07-6

ISSN : 1660-5934

Référence électronique

Diane Duclos, «Eclairage - Des cafés littéraires de Bagdad aux cafés virtuels », Annuaire suisse de politique de développement [En ligne], 27-2 | 2008, mis en ligne le 19 mars 2010, consulté le 08

septembre 2020. URL : http://journals.openedition.org/aspd/171 ; DOI : https://doi.org/10.4000/aspd. 171

(C) The Graduate Institute I Geneva 


\title{
Eclairage \\ Des cafés littéraires de Bagdad aux cafés virtuels
}

\author{
Diane Duclos*
}

D

epuis les années 1960, un grand nombre d'intellectuels irakiens issus de diverses communautés (ethniques et confessionnelles), et de diverses catégories sociales, ont peu à peu quitté un pays où toute forme d'opposition fut progressivement muselée. Pourtant, les débats entamés dans les années 1950 dans les cafés littéraires de Bagdad n'ont jamais été complètement interrompus: de leurs ports d'attache respectifs, peintres, romanciers, poètes, chercheurs, hommes politiques, cinéastes... n'ont cessé de penser leur société d'origine, de la représenter par les mots ou les images, jouissant de la liberté de ton accordée par leur statut d'exilé. Parmi eux, nombreux sont les opposants politiques qui n'ont pu retourner à Bagdad jusqu'en 2003, par crainte de représailles contre eux et leurs proches. Non seulement la chute du régime baathiste n'a pas engendré de mouvement de retour - sinon ponctuels - chez ces Irakiens exilés, mais elle a provoqué une nouvelle vague de départs. Comment des Irakiens ayant quitté leur pays à différentes périodes et vivant dans différentes régions du monde s'organisent-ils de l'extérieur, pour peser sur les débats nationaux irakiens? I/ s'agit ici d'approcher le lien entre migrations et développement non pas exclusivement en termes de transfert de fonds ou de connaissances d'une communauté diasporique vers une société d'origine, mais en termes d'échanges d'idées, en prê- tant une attention particulière aux nouveaux canaux empruntés par cette intelligentsia dispersée pour maintenir une certaine forme de dialogue. Le mode de fonctionnement de la revue Mesopotamia, qui paraît en Irak depuis 2004 et dont les articles sont envoyés via Internet par des Irakiens de l'intérieur et de l'extérieur, peut nous apporter un éclairage intéressant sur ce nouveau "cyberespace de débat", ainsi que sur ses limites.

\section{L'engagement des intellectuels en exil dans la (re)construction de l'Irak}

Les décennies de dictature qu'a connues I'Irak et les images de chaos qui nous parviennent depuis 2003 tendent à nous faire oublier qu' «avant de sombrer sous la dictature, puis devenir le théâtre de la violence, le pays, ou du moins ses principales villes connurent une vie politique intense [...]»1. Tributaire de l'alternance de périodes de relative ouverture et de périodes de répression, cette vie politique était en Irak intimement mêlée aux sphères culturelles et artistiques qui s'épanouissaient dans les nombreux cafés littéraires de Bagdad: on y débattait notamment du meilleur modèle à adopter pour la société irakienne et des dernières innovations en matière de poésie ou de peinture. Dans un article intitulé "Le déclin de I'intelligentsia de gauche en Irak», Salaam Yousif rappelle l'implication des intellectuels

* Etudiante à I'Institut de hautes études internationales et du développement, Genève.

1 H. Bozarslan, "Introduction», L'Irak en perspective, Revue des mondes musulmans et de la méditerranée [en ligne], $n^{0} 117-118,2007$, pp. 13-29, citation p. 15. 
irakiens au sein des mouvements politiques de gauche et plus particulièrement du Parti communiste irakien (PCI) des années 1950 aux années 1970. II cite ainsi certains Irakiens «qui constituaient un leadership intellectuel et moral et qui, à un degré ou un autre, se sont identifiés au PCl ou en ont été proches ${ }^{3}$. Un engagement qui pèsera lourd dans le choix de l'exil. Dès les années 1960, certains membres de cette génération d'intellectuels engagés quittent un pays où l'opposition politique est de plus en plus menacée, ouvrant le chemin de l'exil à des générations d'Irakiens. Les mouvements de départ s'accentuent avec l'intensification de la répression en Irak entre 1978 et 1979. Ces hommes et ces femmes s'organisent rapidement en réseaux à partir de différents pôles géographiques comme Beyrouth, Damas, Amman, Sofia, Prague, Paris, Rome ou Londres, notamment grâce aux antennes du Parti communiste irakien, ou encore à travers un certain nombre de revues littéraires et politiques $^{4}$. Ainsi, depuis Rome, le peintre Jaber Alwan fonde la ligue des artistes, journalistes et écrivains irakiens rassemblant des opposants au régime de Saddam Hussein. De Londres, le chercheur Faleh Abdul Jabar crée le Centre d'études stratégiques irakiennes. A Damas, Fakhri Karim met sur pied la maison d'éditions Dâr Al Madâ... Pendant ce temps, de nombreux Irakiens continuent de fuir un régime autoritaire, un pays en guerre, un pays sous embargo: "Depuis Badr Sakir alSayyab ${ }^{5}$, nombreux furent les hommes de culture irakiens qui vécurent en exil. Certes, ce fut principalement l'expression écrite, la plus répandue et la plus connue, qui fut frappée d'interdits, mais la forte pression politique poussa également de nombreux artistes à vivre à l'étranger. Contrairement à ce qui était le cas au Liban, ces expatriés ne gardèrent pas de contact avec la culture qui se développait à l'intérieur du pays, où souvent ils étaient interdits de séjour. ${ }^{6}$

Si les intellectuels de l'extérieur s'organisent en réseaux, le contexte national irakien rend très difficile la communication avec les intellectuels de l'intérieur. Depuis une dizaine d'années, Internet a largement modifié leurs modes de communication. Cet outil se présente dès son apparition comme un moyen de communication efficace permettant à des personnes du monde entier d'échanger de manière instantanée. A-t-il pour autant permis à ces intellectuels exilés de transcender les frontières et de peser sur le débat de la reconstruction d'un Etat irakien après la chute de Saddam Hussein?

\section{L'émergence et le rôle d'Internet: "des réseaux en recomposition " ?"}

«Au cœur de Londres, face au portail de Kensington Palace, se trouve le Diana's Cafe, fréquenté par les émigrés irakiens, toutes classes sociales et origines ethniques confondues. C'est I'un des hauts lieux irakiens à l'étranger. [...] Pour ma part, je fréquente également un café bien particulier par le réseau Internet: il s'agit d'Iraknet, créé et géré par un Irakien vivant aux Etats-Unis. Ce site comprend un café virtuel, le café

2 Fondé en 1934, le PCl est le plus ancien parti politique irakien. II connaît une première scission en 1967 lorsqu'Aziz al-Hajj crée le PCl-Commandement central. La mouvance kurde du parti s'autonomise quant à elle en 1993 lors du Ve congrès du parti.

3 S. Yousif, "Le déclin de l'intelligentsia de gauche en Irak», L'Irak en perspective, Revue des mondes musulmans et de la Méditerranée, $n^{0}$ 117-118, pp. 51-79, citation p. 54.

4 Certains entretiens ont notamment révélé la participation d'Irakiens à la World Marxist Review ou encore à la revue libanaise Sha'er (poésie) pour faire entendre leur voix.

5 Poète irakien né en 1926 à Jaykour dans le sud de I'Irak, mort en 1964 au Koweït.

6 S. Naef, A la recherche d'une modernité arabe. L'évolution des arts plastiques en Egypte, au Liban et en Irak, Genève, Editions Slatkine, 1996, 450 p., citation p. 206.

7 Voir J.-Ch. Depaule, "Poésies et poètes arabes en réseaux», in F. Mermier et N. Puig (dir.), Itinéraires esthétiques et scènes culturelles au Proche-Orient, Beyrouth, Institut français du Proche-Orient, 2007, pp. 21-44. 
Al-'Azzawî, où les internautes peuvent communiquer entre eux, suivant en cela une longue tradition bagdadienne, puisque les habitants de la capitale avaient pour coutume de se réunir dans les cafés publics pour échanger des idées. $\|^{8}$

S'intéressant aux poètes arabes en réseaux, Jean-Charles Depaule note que "le web" ne remplace pas les modes de circulation en vigueur mais vient les compléter en s'y superposant et, dans certaines circonstances, en les doublant (en les dupliquant et/ou en les dépassant, en les prenant de vitesse), tantôt en relayant certaines séquences ou segments ${ }^{9}$. Internet a-t-il permis, parallèlement au changement de régime en Irak, de renforcer le dialogue entre Irakiens de l'intérieur et de l'extérieur? En 2004, Selim Matar, écrivain irakien résidant à Genève depuis le début des années 1980, crée la revue trimestrielle Mesopotamia10, qui se consacre à la définition de l'identité irakienne en se concentrant sur la diversité de ses composantes: "Elle [Mesopotamia] vise, par le biais de la connaissance mutuelle, à dépasser le fanatisme ethnique, religieux ou idéologique et appelle à la fraternité, la compréhension mutuelle et la tolérance. "11 Tous les articles sont collectés grâce au web et c'est aussi par ce biais que tous les deux numéros, un thème est défini et communiqué. Parmi eux: cinq mille ans de féminité en Irak, cinq mille ans de religiosité irakienne, cinq mille ans d'écriture irakienne ainsi que des numéros spéciaux sur les principales villes du pays. Les numéros à thème paraissent également sous format de livres édités à Beyrouth. Une fois la partie rédactionnelle achevée, les collaborateurs de Bagdad se chargent de la mise en page, de l'impression et de la distribution de la revue dans tout le pays. Toutes ces étapes révèlent le caractère transnational de cette revue, dont le fonctionnement est rendu possible grâce à Internet. Les principaux dossiers thématiques revêtent une dimension historique, dont la formalisation est, selon les fondateurs de la revue, essentielle à l'avènement d'une nation irakienne pacifiée.

La notion d'identité au cœur du projet Mesopotamia est envisagée comme une construction visant à rapprocher les différentes composantes de la population sur la base des frontières territoriales de l'actuel Irak. Ainsi, si la revue se donne pour mission de réconcilier les habitants de l'Irak, elle se heurte rapidement aux revendications des nationalistes kurdes. Faisant suite à différents ouvrages de Selim Matar sur l'identité irakienne, Mesopotamia a placé au centre des discussions le désaccord sur la définition d'une identité et surtout d'un territoire irakiens. Un tel exemple met en lumière l'influence d'Internet sur le travail des intellectuels irakiens en exil, en facilitant notamment la diffusion des idées vers I'Irak et en sollicitant un nouvel espace de débat. II révèle également les spécificités du contexte irakien où violences quotidiennes et occupation sont autant d'obstacles à l'expression des Irakiens. Si Internet permet la diffusion des idées des Irakiens de l'extérieur vers l'intérieur du pays, la participation des intellectuels installés à l'intérieur est en fait limitée: $90 \%$ des personnes qui publient dans cette revue ne résident pas en Irak.

Ainsi, malgré l'exil et la dispersion, les intellectuels irakiens ne cessent de débattre de l'avenir de leur pays et de penser son histoire. Au cœur de leurs discussions virulentes, on retrouve le thème de la construction, ou de la reconstruction d'un Etat irakien. Depuis une dizaine d'années, Internet représente une nouvelle ressource qui,

8 L. Kubba, "Les fantômes de Bagdad", Mémoires d'Irakiens: à la découverte d'une société vaincue..., Monde arabe Maghreb-Machrek, nº 163 (numéro spécial), 1999, pp. 70-74, citation p. 70.

J.-Ch. Depaule, op. cit., p. 40.

10 Voir <http://www.mesopotamia4374.com>.

11 Extrait du dossier de présentation de la revue en vue de l'obtention d'un financement. 
sans abolir les frontières, facilite les échanges et relance dans une certaine mesure le débat entre Irakiens de l'intérieur et Irakiens de l'extérieur. Ces échanges paraissent d'autant plus essentiels dans un pays sous occupation, qui voit ses frontières et ses modes de gouvernance remis en cause.

\section{Bibliographie}

Bozarslan, Hamit, "Introduction», L'Irak en perspective, Revue des mondes musulmans et de la Méditerranée [en ligne], $n^{0} 117-118,2007$, pp. 13-29.

Depaule, Jean-Charles, "Poésies et poètes arabes en réseaux», in Franck Mermier et Nicolas Puig (dir.), Itinéraires esthétiques et scènes culturelles au Proche-Orient, Beyrouth, Institut français du Proche-Orient, 2007, pp. 21-44.

El Janabi, Abdul Kader, Horizon vertical, Arles, Sindbad/Actes Sud, 1998, 114 p.

Kubba, Layth, "Les fantômes de Bagdad", Mémoires d'Irakiens: à la découverte d'une société vaincue..., Monde arabe Maghreb-Machrek, nº 163 (numéro spécial), 1999, pp. 70-74.

Matar, Selim, 1999, "Les séquelles de l'histoire", L'Irak, de Babel à Babel, Oantara, magazine des cultures arabe et méditerranéenne, $\mathrm{n}^{0} 31$, Paris, Institut du monde arabe, pp. 40-43.

Naef, Silvia, A la recherche d'une modernité arabe. L'évolution des arts plastiques en Egypte, au Liban et en Irak, Genève, Editions Slatkine, 1996, $450 \mathrm{p}$.

Yousif, Salaam, 2007, "On the Decline of the Leftist Intelligentsia in Iraq", L'Irak en perspective, Revue des mondes musulmans et de la Méditerranée [en ligne], $n^{0}$ 117-118, pp. 51-79. [La version française de l'article, "Le déclin de l'intelligentsia de gauche en Irak», est publiée dans la revue imprimée.] 